\title{
Frequency and temperature dependence in electromagnetic properties of Martian analog minerals
}

\author{
David Stillman $^{1,2}$ and Gary Olhoeft ${ }^{1}$ \\ Received 1 August 2007; revised 3 February 2008; accepted 28 April 2008; published 13 September 2008.
}

[1] Ground-penetrating radar (GPR) has the potential to image the Martian subsurface to give geological context to drilling targets, investigate stratigraphy, and locate subsurface water. GPR depth of penetration depends strongly on the electromagnetic (EM) properties (complex dielectric permittivity, complex magnetic permeability, and DC resistivity) of the subsurface. These EM properties in turn depend on the mineralogical composition of the subsurface and are sensitive to temperature. In this study, the EM properties of Martian analog samples were measured versus frequency (1 MHz-1 GHz) and at Martian temperatures (180-300 K). Results from the study found the following: gray hematite has a large temperature-dependent dielectric relaxation, magnetite has a temperature-independent magnetic relaxation, and JSC Mars-1 has a broad temperature-dependent dielectric relaxation most likely caused by absorbed water. Two orbital radars, MARSIS and SHARAD, are currently investigating the subsurface of Mars. On the basis of the results of our measurements, the attenuation rate of gray hematite is 0.03 and $0.9 \mathrm{~dB} / \mathrm{m}$, magnetite is 0.04 and $1.1 \mathrm{~dB} / \mathrm{m}$, and JSC Mars- 1 is 0.015 and $0.09 \mathrm{~dB} / \mathrm{m}$ at MARSIS and SHARAD frequencies, respectively, and at the average Martian temperature of $213 \mathrm{~K}$. With respect to using GPR for subsurface investigation on Mars, absorbed water will be a larger attenuator of radar energy as high concentrations of magnetite and gray hematite are not found globally on Mars.

Citation: Stillman, D., and G. Olhoeft (2008), Frequency and temperature dependence in electromagnetic properties of Martian analog minerals, J. Geophys. Res., 113, E09005, doi:10.1029/2007JE002977.

\section{Introduction}

[2] The Mars orbital radars, MARSIS (bandwidth: 1.35.5 MHz) and SHARAD (bandwidth: 15-25 MHz), were designed to receive reflected radar signals from subsurface targets as deep as $5 \mathrm{~km}$ and $1 \mathrm{~km}$, respectively [Safaeinili et al., 2001; Picardi et al., 2005; Seu et al., 2004, 2007]. Outside the polar regions, MARSIS and SHARAD rarely receive deep reflected energy from the Martian subsurface. There are three possible explanations as to why this energy is not returning from soil and rock covered areas. First, there may be no distinct changes in electrical and/or magnetic properties to cause radar energy to be reflected. Second, there may be significant changes in electrical and/or magnetic properties in the shallow subsurface, which scatters the majority of the radar energy [Grimm et al., 2006]. Lastly, dielectric and magnetic relaxations of Martian subsurface minerals may be attenuating the radar energy. This last theory is the focus of this paper.

\footnotetext{
${ }^{1}$ Department of Geophysics, Colorado School of Mines, Golden, Colorado, USA.

${ }^{2}$ Now at Department of Space Studies, Southwest Research Institute, Boulder, Colorado, USA.

Copyright 2008 by the American Geophysical Union. 0148-0227/08/2007JE002977\$09.00
}

[3] The magnitude of radar energy that is attenuated as it propagates through a material depends on the material's EM properties (DC conductivity, complex dielectric permittivity, and complex magnetic permeability). On Earth, the largest sources of attenuation are usually due to conductive and dielectric relaxation losses that are caused by liquid water and clays. On Mars, near surface liquid water is unlikely due to the cold temperatures and clays are less abundant. Magnetic relaxations rarely occur on Earth because of the lack of ferromagnetic and ferrimagnetic minerals in Earth soils, however these relaxations have proven to create significant radar losses [Olhoeft and Capron, 1993, 1994]. Unlike Earth, Mars is known to contain an abundance of ferrimagnetic minerals at its surface [Hargraves et al., 1979, 2000]. In fact, every particle of the Martian global windblown dust layer is magnetic at DC (zero) frequency and is believed to be composed of about $2 \%$ magnetite [Bertelsen et al., 2004]. Consequently, magnetic and dielectric relaxation losses on Mars may be the dominant radar loss mechanisms and therefore must be considered when predicting GPR depth of penetration.

[4] EM properties can change as a function of temperature [Olhoeft, 1976; Dunlop and Özdemir, 1997]. Mars experiences a large range of daily global temperature fluctuations (154-300 K) with an average annual surface temperature ranging from $154-218 \mathrm{~K}$ as a function of latitude [Clifford, 1993]. Because of the large temperature 
fluctuations on Mars, these temperature-dependent properties will change as a function of the time of day and have different values than those measured in the typical Earth environment. Consequently, measurements of EM properties (dielectric permittivity, magnetic permeability, and DC conductivity) made at room temperature $(\approx 298 \mathrm{~K})$ are not representative of Mars.

[5] The purpose of this study was to measure the EM properties of dry Martian analog minerals in the Martian environment to determine whether these minerals cause significant attenuation at radar frequencies.

\section{Background}

\subsection{EM Properties}

[6] The EM properties (DC conductivity, $\sigma_{D C}$, complex dielectric permittivity, $\varepsilon^{*}$, and complex magnetic permeability, $\mu^{*}$ ) of a material affect how EM energy propagates and attenuates through the material. The DC conductivity represents the ability of free charge to flow under the presence of a static electric field. Dielectric permittivity represents the ability of bound charges to separate under the presence of an electric field. Magnetic permeability represents the ability of magnetic moments to align with a magnetic field. The complex dielectric permittivity, $\varepsilon^{*}$, and complex magnetic permeability, $\mu^{*}$, are defined as

$$
\begin{gathered}
\varepsilon^{*}=\varepsilon_{o} \varepsilon_{r}^{*}=\varepsilon_{o}\left(\varepsilon_{r}^{\prime}-i \varepsilon_{r}^{\prime \prime}\right), \\
\mu^{*}=\mu_{o} \mu_{r}^{*}=\mu_{o}\left(\mu_{r}^{\prime}-i \mu_{r}^{\prime \prime}\right),
\end{gathered}
$$

where $\varepsilon_{o}$ is the dielectric permittivity of vacuum $(8.8541 \times$ $\left.10^{-12} \mathrm{~F} / \mathrm{m}\right), \varepsilon_{r}^{*}$ is the complex relative dielectric permittivity, $\varepsilon_{r}^{\prime}$ is the real part of the relative dielectric permittivity, $\varepsilon_{r}^{\prime \prime}$ is the imaginary part of the relative dielectric permittivity, $\mu_{o}$ is the magnetic permeability of vacuum $\left(4 \pi \times 10^{-7} \mathrm{H} / \mathrm{m}\right)$, $\mu_{r}^{*}$ is the complex relative magnetic permeability, $\mu_{r}^{\prime}$ is the real part of the relative magnetic permeability, and $\mu_{r}^{\prime \prime}$ is the imaginary part of the relative magnetic permeability. The real parts of $\varepsilon_{r}^{*}$ and $\mu_{r}^{*}$ represent the amount of energy stored, while the imaginary parts represent the amount of energy lost.

[7] Frequency dependence of dielectric permittivity occurs because charge separation does not occur instantaneously. Charges separate with finite velocities, thus if the external field is reversing polarity too quickly the charges cannot move fast enough to keep up. The time it takes for the charges to align from one polarity of the external electric field to the next, is twice the time constant of relaxation, $\tau$. The relaxation frequency, $f_{r}$, is a function of $\tau$ and is defined as

$$
f_{r}=\frac{1}{2 \pi \tau}
$$

If the frequency of the external field is much less than the relaxation frequency, then the charges will have enough time to fully separate before the external field switches polarity. However, if the frequency of the external field is much greater than the relaxation frequency, then the charges will not have enough time to fully separate and no charge separation takes place. If the frequency of the external field is near the relaxation frequency, the charges are in constant motion and the internal electric field is out of phase with the external electric field. The constant motion of charges results in energy loss as kinetic energy is converted into thermal energy of the material through momentum transfer (collisions and/or electromagnetic interactions). Consequently, the maximum energy loss occurs at the relaxation frequency because the charges are in constant motion at the maximum separation distance.

[8] Likewise, frequency dependence of magnetic permeability occurs when the magnetic moments of a material can no longer realign parallel to an external magnetic field before the field switches direction. Frequency dependence of dielectric permittivity and magnetic permeability can be modeled using the Cole-Cole equation [Cole and Cole, 1941]:

$$
X^{*}=X^{\prime}-i X^{\prime \prime}=X \propto+\frac{X_{D C}-X_{\propto}}{1+(i \omega \tau)^{\alpha}}
$$

where $X$ is the relative dielectric permittivity or relative magnetic permeability, $X^{*}$ is the complex of $X, X^{\prime}$ is the real part of $X, X^{\prime \prime}$ is the imaginary part of $X, X_{\infty}$ is the highfrequency limit of $X, X_{D C}$ is the low-frequency limit of $X, \omega$ is the angular frequency (radians/second), $\tau$ is the time constant of relaxation (seconds), and $\alpha$ is the Cole-Cole distribution parameter. The Cole-Cole equation assumes a log-normal distribution of the time constants of relaxation, $\tau$. The log-normal distribution is described by the Cole-Cole distribution parameter, $\alpha$, and the mode of the distribution is the time constant of relaxation [Cole and Cole, 1941]. If the Cole-Cole distribution parameter, $\alpha$, is unity, then there is a single time constant of relaxation and the Cole-Cole equation reduces to the Debye equation [Debye, 1929]. Typically, the distribution parameter is equal to unity in gases because they are perfectly homogeneous. Any heterogeneity in crystal structure or grain size will cause a soil sample to have a distribution parameter less than unity.

[9] Kauzmann [1942] demonstrated that the generalized Boltzmann temperature dependence could be used to predict how the time constant of relaxation, $\tau$, changes as a function of temperature:

$$
\tau=\tau_{\infty} e^{\frac{E}{k T}}
$$

where $\tau_{\infty}$ is the time constant of relaxation at infinite temperature (seconds), $E$ is the activation energy $(\mathrm{eV}), k$ is Boltzmann's constant $\left(8.6176 \times 10^{-5} \mathrm{eV} / \mathrm{K}\right)$, and $T$ is the temperature $(\mathrm{K})$. The activation energy, $E$, represents an energy barrier that must be overcome in order for the charges to fully separate or for the magnetic moments to align. As temperature increases, the charges and magnetic moments have more energy, thus making it easier to overcome this energy barrier. Therefore, as temperature is increased, the time constant of relaxation shifts to a smaller period.

[10] The Néel model (equation (6)) is a form of the generalized Boltzmann temperature dependence model that is specific for the time constant of relaxation for magnetic permeability [Néel, 1949]. In this model, the activation 
energy is a function of particle volume, saturation magnetization, and coercivity [Dunlop and $\ddot{O} z$ demir, 1997]

$$
\tau=\frac{\tau_{o}}{2} e^{\frac{\mu_{o} v M_{s} H_{c}}{2 k T}}
$$

where $\tau_{o} \approx 10^{-9} \mathrm{~s}$ is the atomic reorganization time or interval between successive thermal excitations, $v$ is the magnetic grain volume $\left(\mathrm{m}^{3}\right), H_{c}$ is the coercivity $(\mathrm{A} / \mathrm{m}), M_{s}$ is the saturation remnance $(\mathrm{A} / \mathrm{m})$, and $k$ is Boltzmann's constant $\left(1.38065 \times 10^{-23} \mathrm{~J} / \mathrm{K}\right)$.

[11] To model both the temperature and frequency dependence of a sample, the generalized Boltzmann temperature dependence can be inserted into the Cole-Cole equation

$$
X_{r}^{*}=X_{r}^{\prime}-i X_{r}^{\prime \prime}=X_{\infty}+\frac{X_{D C}-X_{\infty}}{1+\left(i \omega \tau_{\infty} e^{E / k T}\right)^{\alpha}}
$$

This equation can then be used to model the EM properties of a material at any temperature and frequency.

[12] Another important factor to consider when comparing lab values to field values is the soil's density. Since the dielectric permittivity and magnetic permeability vary as a function of density, they were normalized to a bulk density of $1.60 \mathrm{~g} / \mathrm{cm}^{3}$. The high-frequency limit of the relative dielectric permittivity, or electronic polarization, can be found using a Lichtenecker power law mixing formula [Olhoeft and Strangway, 1975]:

$$
\varepsilon_{\infty}=(K)^{d}=(1.93)^{d}
$$

where $\varepsilon_{\infty}$ is the high-frequency limit of the real part of the relative dielectric permittivity, $K$ is the mode of the highfrequency limit of 114 lunar samples, 261 pure minerals, and 367 rocks (1.93 \pm 0.17$)$ [Olhoeft and Strangway, 1975], and $d$ is the bulk density $\left(\mathrm{g} / \mathrm{cm}^{3}\right)$.

[13] Density corrections for magnetic permeability are more difficult than density corrections for dielectric permittivity because magnetic particles interact with each other. The most commonly used magnetic permeability mixing law was empirically derived [Strangway, 1967]

$$
\mu_{r}^{*}=\frac{\mu_{r}^{* M}-1}{2 V-\mu_{r}^{* M} V+\mu_{r}^{* M}-1} V^{2}+1
$$

where $\mu_{r}^{* M}$ is the real part of the relative magnetic permeability at a volume of $100 \%$, and $V$ is the volume $\left(\mathrm{cm}^{3}\right)$.

[14] Once the EM properties of a material are known, they can be used to calculate the amount of loss they will create. This is done by splitting the wave number into its real and imaginary parts:

$$
k^{2}=\omega^{2} \mu^{*} \varepsilon^{*}-i \omega \mu^{*} \sigma_{D C}=(\beta-i \alpha)^{2},
$$

where the phase parameter, $\beta$, is the real part of the wave number and represents how much energy is stored in a material as the EM field passes through it, and the attenuation parameter, $\alpha$, is the imaginary part of the wave number and represents how much energy is attenuated in a material as the EM field passes through it. Assuming a complex magnetic permeability, complex dielectric permittivity, and DC conductivity, the attenuation parameter equals

$$
\alpha=\frac{\omega}{c} \sqrt{\frac{\sqrt{A^{2}+B^{2}}-A}{2}},
$$
where: $A=\mu_{r}^{\prime} \varepsilon_{r}^{\prime}-\mu_{r}^{\prime \prime}\left(\varepsilon_{r}^{\prime \prime}+\frac{\sigma}{\omega \varepsilon_{o}}\right), B=\mu_{r}^{\prime \prime} \varepsilon_{r}^{\prime}+\mu_{r}^{\prime}\left(\varepsilon_{r}^{\prime \prime}+\frac{\sigma}{\omega \varepsilon_{o}}\right)$
and $c=\frac{1}{\sqrt{\varepsilon_{o} \mu}}$.

[15] The attenuation parameter, $\alpha$, is then converted from nepers per meter into an attenuation rate, $\eta$, with units of decibels per meter (equation (12)). The maximum depth of penetration can then be found by dividing the dynamic range of the radar system by twice the attenuation rate (equation (13)). This is defined as the maximum depth of penetration because only EM losses have been included. The addition of other loss mechanisms such as scattering and geometrical spreading would further reduce the depth of penetration. The dynamic range of SHARAD and MARSIS is estimated to be $30-50 \mathrm{~dB}$ in the ground [Safaeinili et al., 2001; Picardi et al., 2005; Seu et al., 2004, 2007]. The upper limit of $50 \mathrm{~dB}$ was used for calculations in this paper.

$$
\begin{gathered}
\qquad \eta=20 \log _{10}\left(e^{\alpha}\right)=8.686 \alpha \\
\text { Maximum Depth of Penetration }=\frac{\text { Dynamic Range }}{2 \eta}
\end{gathered}
$$

[16] As shown in equation (11), the attenuation parameter, $\alpha$, varies proportionally with frequency. To better illustrate the attenuation caused by the EM properties of the sample, loss tangent graphs will be used in this paper. The loss tangent, $\tan \delta$, represents the EM energy lost per cycle divided by the energy stored per cycle [Ward and Hohmann, 1988; Grimnes and Martinsen, 2000]. The conduction, dielectric, and magnetic loss tangent are defined as:

$$
\begin{gathered}
\tan \delta_{C}=\frac{\sigma_{D C}}{\omega \varepsilon_{0} \varepsilon^{\prime}}, \\
\tan \delta_{D}=\frac{\varepsilon_{r}^{\prime \prime}}{\varepsilon_{r}^{\prime}}, \\
\tan \delta_{M}=\frac{\mu_{r}^{\prime \prime}}{\mu_{r}^{\prime}},
\end{gathered}
$$

The total electrical loss tangent is defined as

$$
\tan \delta_{E}=\tan \delta_{C}+\tan \delta_{D}
$$

[17] Since the DC conductivity of the dry Martian analog samples was less than the detection limit of the apparatus $\left(6.67 \times 10^{-5} \mathrm{mho} / \mathrm{m}\right)$ [Stillman, 2006], the conductive loss tangent can be neglected. The total EM loss tangent is

$$
\tan \delta_{E M}=\tan \left(\frac{\delta_{E}+\delta_{M}}{2}\right)=\frac{\alpha}{\beta} .
$$

\subsection{Previous Measurements}

[18] The magnetic properties of Mars at DC frequencies have been studied by making in-situ measurements. These measurements were made by attaching magnets on every 
Table 1. Major ( $>20 \%)$ and Minor Mineralogy of the Samples Determined by $X_{R D}{ }^{a}$

\begin{tabular}{clll}
\hline Sample Name & \multicolumn{1}{c}{ Location } & Major Mineralogy & Minor Mineralogy \\
\hline FeOx & synthetic ferric oxide & Hem & Goe \\
GH-1 & Keweenaw Peninsula, Michigan & GH & Mag, Q \\
GH-2 & Champion Mine Dump, Michigan & GH & Mag \\
JSC-1 & Pu'u Nene, Hawaii & Plag & And, Q \\
Mag-1 & Champion Mine Dump, Michigan & Mag & Hem, Q \\
Mag-2 & Peru & Mag, Mag & Hem, Ill, Q, Plag \\
Mag-3 & near Yuma, AZ & Maghem, Hem & \\
Maghem & near Kirov Rog, Russia & Njaro & \\
Njaro & near Zhuravlinskogo, Russia & Fo & Fa \\
Oliv & Green Sand Beach, Hawaii & Plag & Mag \\
Plag & Pu'u Nene, Hawaii & Q & \\
Sand & Ottawa, Illinois & & \\
\hline
\end{tabular}

Plag, plagioclase feldspar (anorthite/albite series); Mag, magnetite; GH, gray hematite; Goe, goethite; Q, quartz; And, andalusite; Hem, red hematite; Illm, illmenite; Maghem, maghemite; Njaro, natrojarosite; Fa, fayalite; Fo, forsterite.

${ }^{a}$ Since XRD cannot distinguish between gray and red hematite, these distinctions were made by the color of the sample.

Martian lander and by measuring the remanent magnetic field from Martian orbiters. The in situ measurements have found that Martian rocks, soils, and dust contain significantly more magnetic minerals than Earth, and the Martian global dust layer has an average saturation magnetization of 1-4 $\mathrm{Am}^{2} / \mathrm{kg}$ and a density magnetic susceptibility of $9-33 \times 10^{-6} \mathrm{~m}^{3} / \mathrm{kg}$ [Morris et al., 2001]. While the domain type is unknown the mineral causing this magnetization is either magnetite or titanomagnetite [Morris et al., 2004; Bertelsen et al., 2004; Goetz et al., 2005; Yen et al., 2005]. In places the Martian remanent magnetic field that is 10 times greater than Earth's remanent magnetic field and is most likely caused by thermoremanent magnetization of singledomain magnetite or titanomagnetite [Dunlop and ArkaniHamed, 2005].

[19] Laboratory measurements of the EM properties of Martian analogs at radar frequencies have been made in the past [Olhoeft and Strangway, 1974; Olhoeft and Capron, 1993, 1994; Leuschen, 1999; Heggy et al., 2001, 2003; Heggy and Pommerol, 2005; Williams and Greeley, 2004; Pettinelli et al., 2005]. A brief discussion of these measurements follows.

[20] Olhoeft and Strangway [1974] predicted that the electrical properties of the Martian subsurface would be similar to the Moon, even though the Martian atmosphere contains a small amount of water. This is because small amounts of water (less than seven monolayers) absorbed in the soil do not affect dielectric permittivity at high frequencies [McIntosh, 1966; Olhoeft and Strangway, 1974]. The Martian water would also typically be in the form of ice, thus reducing its effects further. Other than the water/ice transition, Olhoeft and Strangway [1974] state that temperature has no effect on the electrical properties of the Moon soils and therefore should not have an influence on Martian soils. However, Olhoeft [1976] later demonstrated that temperature does have an effect on electrical properties of dry soils. Olhoeft and Strangway [1974] make no mention of magnetic properties of the Martian subsurface. However, Olhoeft and Capron [1993, 1994] found that magnetic soils can have a magnetic relaxation that is the dominant loss mechanism in the soil. This suggests that magnetic losses could be the dominant loss mechanism in the Martian subsurface.

[21] Leuschen [1999] conducted measurements with a vector network analyzer (VNA) using a slotted line for the sample holder. These measurements were made from 10 MHz to $1 \mathrm{GHz}$ and found that JSC Mars-1 has a frequencydependent dielectric permittivity and a frequency-dependent magnetic permeability. Numerous measurements of JSC Mars-1 were conducted in this study and a magnetic permeability above one was never recorded.

Table 2. EM Properties for Samples That Have No Measurable EM Losses ${ }^{\mathrm{a}}$

\begin{tabular}{lcccc}
\hline Sample & Density $(\mathrm{g} / \mathrm{cc})$ & $\begin{array}{c}\text { Real Part of the Relative } \\
\text { Dielectric Permittivity }\left(\varepsilon_{\mathrm{r}}\right)\end{array}$ & $\begin{array}{c}\text { DC Resistivity, } \\
\sigma_{\mathrm{DC}}(\mathrm{k} \Omega \mathrm{m})\end{array}$ & $\begin{array}{c}\text { Real Part of the Relative } \\
\text { Magnetic Permeability }\left(\mu_{\mathrm{r}}\right)\end{array}$ \\
\hline Sand & 1.47 & $2.57 \pm 0.01$ & $>15$ & $1.00 \pm 0.02$ \\
& 1.60 & $2.80 \pm 0.01$ & $>15$ & $1.00 \pm 0.02$ \\
Njaro & 1.39 & $3.07 \pm 0.02$ & $>15$ & $1.00 \pm 0.02$ \\
& 1.60 & $3.52 \pm 0.02$ & & $1.00 \pm 0.02$ \\
FeOx & 0.68 & $1.70 \pm 0.02$ & $>15$ & $1.00 \pm 0.02$ \\
& 1.60 & $3.10 \pm 0.04$ & $>15$ & $1.00 \pm 0.02$ \\
Oliv & 2.00 & & $>15$ & $1.00 \pm 0.02$ \\
& 1.60 & $3.61 \pm 0.03$ & & $1.00 \pm 0.02$ \\
Maghem & 1.14 & $2.78 \pm 0.04$ & $>15$ & $1.28 \pm 0.02$ \\
& 1.60 & $2.41 \pm 0.02$ & $>15$ & $\mathrm{NA}$ \\
\hline
\end{tabular}

${ }^{a}$ The first row shows the modeled results of the measured data. In the second row, the modeled data were corrected for density using a Lichtenecker power law mixing formula. The magnetic permeability of maghemite could not be estimated at a density of $1.60 \mathrm{~g} / \mathrm{cc}$ because the concentration of maghemite in the sample is unknown. 

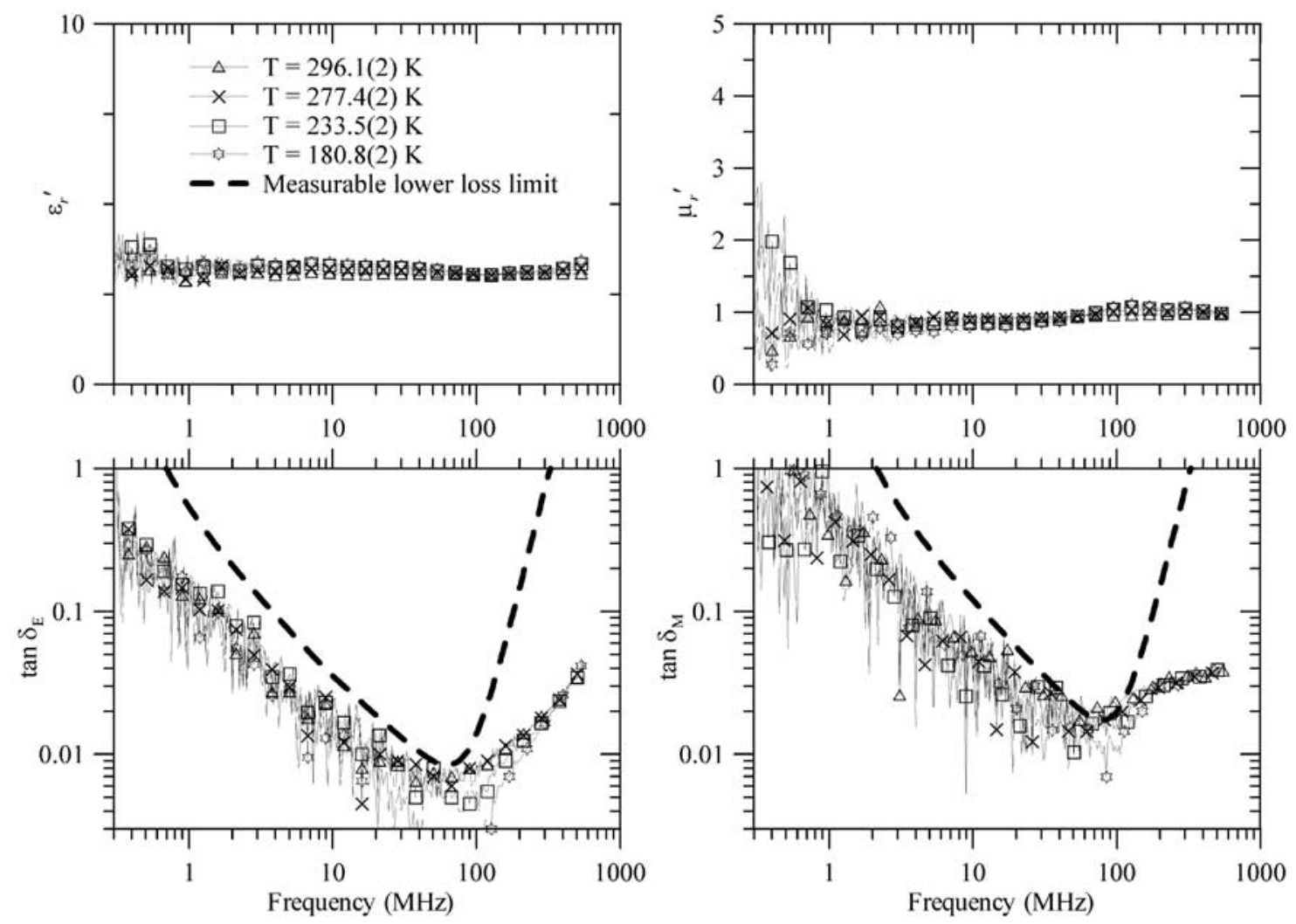

Figure 1. EM properties of Njaro which contain no measurable EM losses and no measurable frequency and temperature dependence. Symbols are located at every tenth data point. Measurement errors are much smaller than the symbols, except for the portion of the data that is below the measurable lower loss limit and in the $\mu_{r}^{\prime}$ at frequencies below $1 \mathrm{MHz}$.

[22] Heggy et al. [2001, 2003] and Heggy and Pommerol [2005] conducted measurements of dielectric permittivity versus frequency for Martian analogs with impedance analyzers. Impedance analyzers cannot measure phase as accurately as the VNA, thus VNAs can measure lower losses than impedance analyzers. Heggy et al. [2001] and Heggy and Pommerol [2005] did not report any magnetic permeability measurements.

[23] Williams and Greeley [2004] measured the complex dielectric permittivity of JSC Mars-1 and Carbondale red clay from $200-1300 \mathrm{MHz}$ at room temperature. Exact details of these measurements were not discussed. They also made microwave transmission measurements on the same samples over a frequency range from 500-12,000 MHz. They assumed no magnetic losses and a magnetic permeability of one to calculate an attenuation rate.

[24] Pettinelli et al. [2005] conducted measurements of two magnetite samples with an LCR (impedance-capacitance-resistance) meter from $500 \mathrm{~Hz}-1 \mathrm{MHz}$ and time domain reflectometry (TDR) from 1-500 MHz. The LCR meter was able to measure both complex dielectric permittivity and complex magnetic permeability because two different sample holders were used to measure each separately. However, these measurements were used to constrain the low-frequency limit of both the dielectric permittivity and magnetic permeability. The TDR measurements are sensitive to the EM velocity of a material. Therefore TDR measurements cannot uniquely measure complex dielectric permittivity and complex magnetic permeability. However, the TDR measurements showed that the EM velocity did not change from $1-500 \mathrm{MHz}$.

[25] All of these previous measurements were conducted at room temperature $(\approx 298 \mathrm{~K})$. The surface temperature on Mars rarely reaches $298 \mathrm{~K}$. Temperature must be accounted for when measuring the EM properties of Martian analogs since EM properties can vary as a function of temperature. Research done by Iben et al. [1996] and Morris et al. [1997] have shown that the electrical properties of some Martian analogs also change as a function of temperature. Iben et al. [1996] observed that both magnetite and red hematite have a temperature-dependent dielectric relaxation centered at 200 and $10 \mathrm{~Hz}$, respectively, at $293 \mathrm{~K}$. Morris et al. [2001] observed that the reflectivity spectrum between 4.62 and $5.45 \mathrm{THz}(650$ and $550 \mathrm{~nm})$ is temperature dependent in a red hematite powder. These previous studies suggest that powdered red hematite is temperature dependent at very high frequencies in the EM spectrum, while magnetite and red hematite are temperature dependent at very low frequencies in the EM spectrum. Consequently, the EM properties of these minerals could be temperature dependent at radar frequencies.

\section{Sample Description}

[26] Martian analogs were selected for their likelihood of being present on Mars and the probability that they possess dielectric and/or magnetic losses (Table 1). Consequently, many of the selected samples were ferrimagnetic, since only 


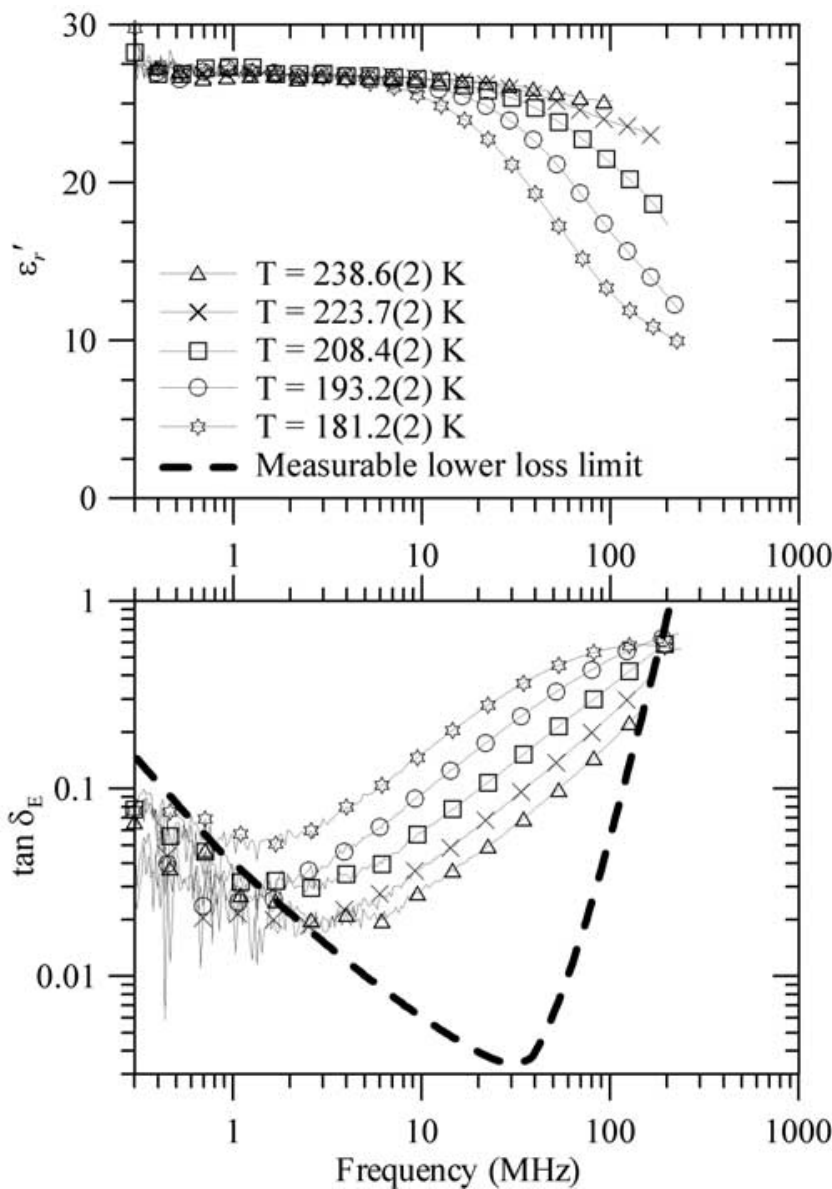

Figure 2. Temperature-dependent dielectric relaxation of GH-1. Symbols are located at every tenth data point. Measurement errors are much smaller than the symbols, except for the portion of the data that is below the measurable lower loss limit.

ferromagnetic or ferrimagnetic minerals possess magnetic losses. All of the samples were measured in a soil form. When only rocks of specific samples could be found, they were crushed into a soil using a nonmetallic mortar and pestle. Each sample was then vacuum dried before it was measured. Vacuum drying is necessary because soils on the surface of Mars are extremely dry and the presence of water can significantly change the dielectric frequency dependence and the DC conductivity of the sample [Olhoeft and Strangway, 1975]. Martian analog samples that were selected for this study included: magnetite, maghemite, gray (coarse grained) hematite, red (fine grained) hematite, olivine, jarosite, and silica sand. A summary justification for each selected Martian analog sample is provided below.

[27] The Mössbauer spectroscopy measurements made by both Spirit (MER-A) and Opportunity (MER-B) confirmed that magnetite $\left(\mathrm{Fe}_{3} \mathrm{O}_{4}\right)$ is the mineral causing the magnetic properties of the Martian dust [Morris et al., 2004; Bertelsen et al., 2004; Madsen et al., 2005; Goetz et al., 2005; Yen et al., 2005]. Not only is magnetite in the dust layer, but it has also been found in basaltic rocks at Gusev crater. It is also the most likely mineral causing the remanent magnetic field of Mars [Dunlop and Arkani-Hamed, 2005]. The percentage of magnetite in the global Martian dust layer is about $2 \%$ by volume [Morris et al., 2004; Bertelsen et al., 2004; Madsen et al., 2005; Goetz et al., 2005; Yen et al., 2005], while the crust has been estimated at $0.2-0.4 \%$ by volume [Dunlop and Arkani-Hamed, 2005]. The estimation of the crust only includes single domain magnetite, therefore the total magnetite concentration may be larger. The domain type of the magnetite in the dust remains unknown.

[28] Nanocrystalline red hematite $\left(\alpha-\mathrm{Fe}_{2} \mathrm{O}_{3}\right)$ or maghemite $\left(\gamma-\mathrm{Fe}_{2} \mathrm{O}_{3}\right)$ may be the mineral causing the anhydrous ferric oxide signature observed by the OMEGA spectrometer onboard Mars Express in the bright areas on Mars [Bibring et al., 2006]. Fine-grained $(<10 \mu \mathrm{m})$ red hematite is also a major component of the homogenous dust on Mars and gives Mars its color [Goetz et al., 2005]. Therefore a synthetic red hematite was measured. Since maghemite is a ferrimagnetic mineral and could possess significant magnetic relaxation losses, it was measured.

[29] Coarse-grained $(>10 \mu \mathrm{m})$ gray hematite $\left(\alpha-\mathrm{Fe}_{2} \mathrm{O}_{3}\right)$ has been spectroscopically identified in three different Martian locations [Christensen et al., 2001]. Opportunity (MER-B) determined that the spectroscopic gray hematite signal at Meridiani Planum was caused by gray hematite concretions that are believed to have precipitated from ironrich groundwater [Squyres and Knoll, 2005].

[30] Johnson Space Center selected JSC Mars-1 as the best Martian analog on Earth because of its similar spectral and magnetic properties to Martian soil [Allen et al., 1997, 1998a, 1998b; Allen and Morris, 1999]. However, JSC Mars-1 is not a perfect Martian analog because it does not contain enough hematite, it contains too much magnetite [Hargraves et al., 1999], and it contains particles that are nonmagnetic. JSC Mars-1 was mined from Pu'u Nene cinder cone, Hawaii [Allen et al., 1997]. The un-oxidized layer directly beneath the JSC Mars-1 layer at Pu'u Nene was also collected (Plag). Both samples are composed mostly of plagioclase feldspar. Plagioclase feldspar is not a mineral type, but rather a combination of two minerals: albite $\left(\mathrm{NaAlSi}_{3} \mathrm{O}_{8}\right)$ and anorthite $\left(\mathrm{CaAl}_{2} \mathrm{Si}_{2} \mathrm{O}_{8}\right)$. About $60 \%$ (by volume) of the Earth's continental crust is composed of plagioclase feldspar as it is a major component of both intrusive and extrusive igneous rocks [Chernicoff and Venkatakrishnan, 1995]. The Martian surface is dominated by igneous flows, and spectral models of both surface type 1 (basalt) and type 2 (weathered basalt or andesite) have estimated plagioclase feldspar content to be between 30 $60 \%$ by volume [Wyatt and McSween, 2002].

[31] The last three samples selected were olivine, jarosite, and silica sand. Olivine $\left[(\mathrm{Mg}, \mathrm{Fe})_{2} \mathrm{SiO}_{4}\right]$ was measured since it has been spectroscopically identified globally [Hoefen et al., 2003] and is a mineralogical component of the dust [Goetz et al., 2005]. Jarosite [(K, Na) $\left(\mathrm{Fe}, \mathrm{Al}_{3}\left(\mathrm{SO}_{4}\right)_{2}(\mathrm{OH})_{6}\right]$ was selected because Opportunity's (MER-B) Mössbauer instrument detected jarosite in the bedrock at Meridiani Planum [Klingelhöfer et al., 2004]. Silica sand $\left(\mathrm{SiO}_{2}\right)$ was selected as a measurement standard since the EM properties of sand are known to be frequency and temperature independent at radar frequencies.

\section{Measurement Apparatus}

[32] Network analyzers have been used to acquire highfrequency electromagnetic (EM) measurements since the 

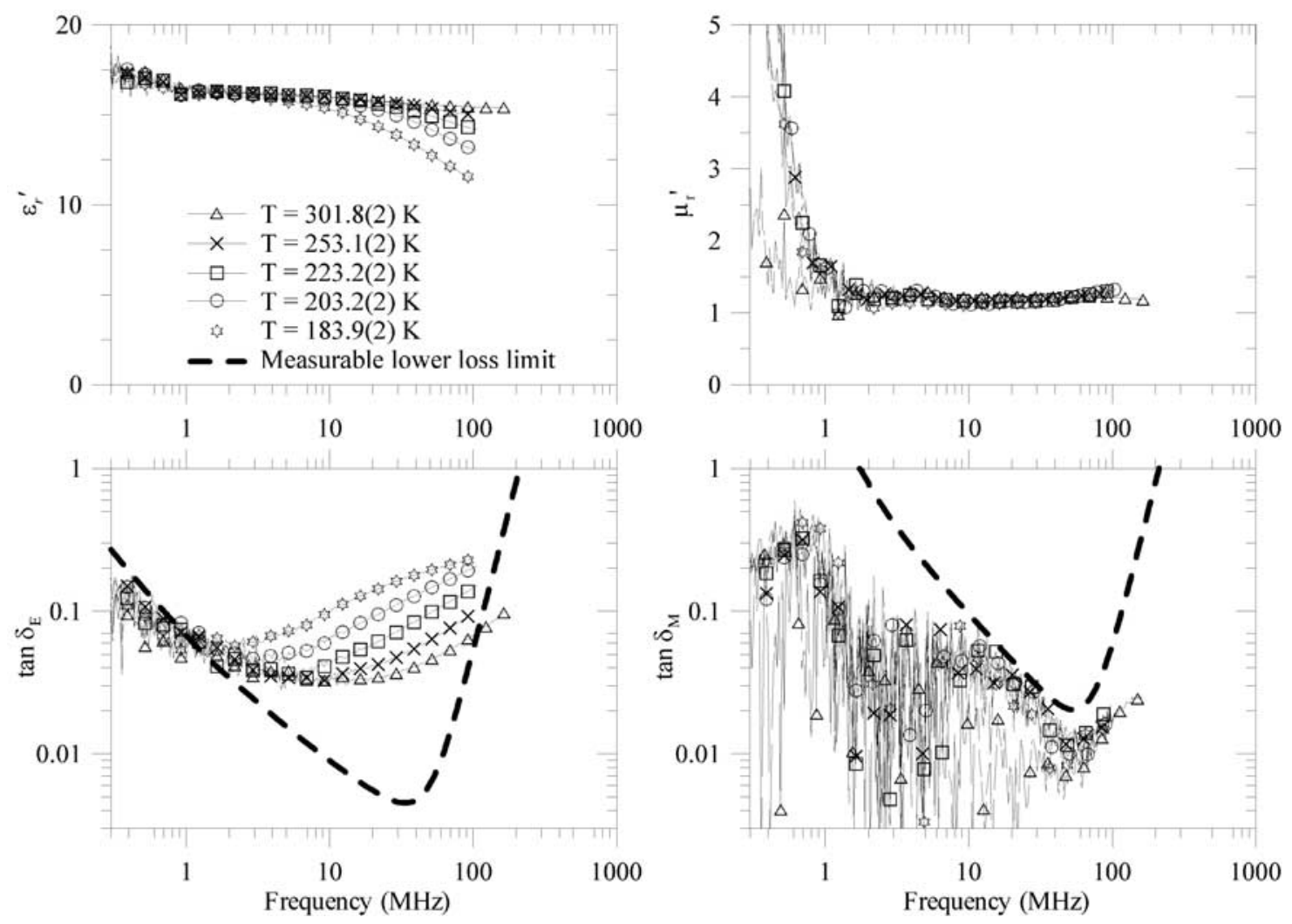

Figure 3. Temperature-dependent dielectric relaxation of GH-2. Symbols are located at every tenth data point. Measurement errors are much smaller than the symbols, except for the portion of the data that is below the measurable lower loss limit and in the $\mu_{r}^{\prime}$ at frequencies below $1 \mathrm{MHz}$.

1960s. In this study, a HP8753D vector network analyzer (VNA) was controlled by a computer with custom software [Stillman, 2006]. The two ports on the VNA were attached to the $14 \mathrm{~mm}$ diameter waveguide sample holder by two phase matched cables and adapters. The complex dielectric permittivity and complex magnetic permeability can be uniquely determined by measuring the scattering parameters of the sample filled sample holder [Adams, 1969; Baker-Jarvis et al., 1993; Stillman, 2006].

[33] Prior to measuring a sample, the VNA and associated cable connections had to be calibrated. A 12 term two port calibration was used to determine the electrical length, dynamic range of the system, and crosstalk or leakage between the ports [Stillman, 2006]. This calibration was performed each time a new sample was measured. Even after calibrating, the data were affected by sample holder resonance and by the finite precision of the VNA. Sample holder resonance occurs when the EM wave approaches half wavelength multiples of the sample holder length [Stillman, 2006]

$$
f_{\text {res }}=\frac{\sqrt{2 c}}{\frac{2 L}{n} \sqrt{\sqrt{\left(\mu_{r}^{\prime} \varepsilon_{r}^{\prime}-\varepsilon_{r}^{\prime \prime} \mu_{r}^{\prime \prime}\right)^{2}+\left(\mu_{r}^{\prime \prime} \varepsilon_{r}^{\prime}+\mu_{r}^{\prime} \varepsilon_{r}^{\prime \prime}\right)^{2}}+\varepsilon_{r}^{\prime} \mu_{r}^{\prime}-\varepsilon_{r}^{\prime \prime} \mu_{r}^{\prime \prime}}},
$$

where $\mathrm{L}$ is the sample holder length $(\mathrm{m})$ and $\mathrm{n}$ is the number of harmonics 1,2 , 3 , etc. The data were truncated after the first harmonic of the resonance frequency, $f_{\text {res }}$.
[34] At lower frequencies, the precision of the measurement is significantly reduced because the HP8753D can only measure phase to a precision of $0.01^{\circ}$ [HewlettPackard, 1994]. When the wavelength of the EM energy is significantly greater than the length of the sample holder, only minute changes $\left(<0.01^{\circ}\right)$ in phase occur. Consequently, the VNA cannot measure the EM properties of a sample at low frequencies as precisely as it can at high frequency. This also creates a measurable lower loss limit for the loss tangent. Therefore the measurable lower loss limit represents the noise floor of the system, thus only data above the measurable lower loss limit were used for modeling. To slightly vary the measurable frequency range $3 \mathrm{~cm}$ (sample volume of $3.7 \mathrm{cc}$ ) and $10 \mathrm{~cm}$ (sample volume of $12.5 \mathrm{cc}$ ) sample holders were used.

[35] To acquire measurements as a function of temperature, the sample holder was placed in an insulated So-Low Ultra-Low freezer (model \#C85-5). The temperature was varied from $180-300 \mathrm{~K}$ with VNA measurements made at intervals of $5-20 \mathrm{~K}$. Computer fans were used to provide circulation inside the freezer to maintain a uniform temperature. The lowest temperature measurement was obtained by increasing the amount of insulation inside the freezer, using an air conditioner to maintain a cool room temperature outside the freezer, and turning off all circulating fans in the freezer so that the coldest/densest air sank to the bottom of the freezer where the measurements were being made. Three YSI 44006 epoxy-encapsulated thermistors were placed inside the freezer: one at the bottom of the freezer, one on the outside of the sample holder, and one 

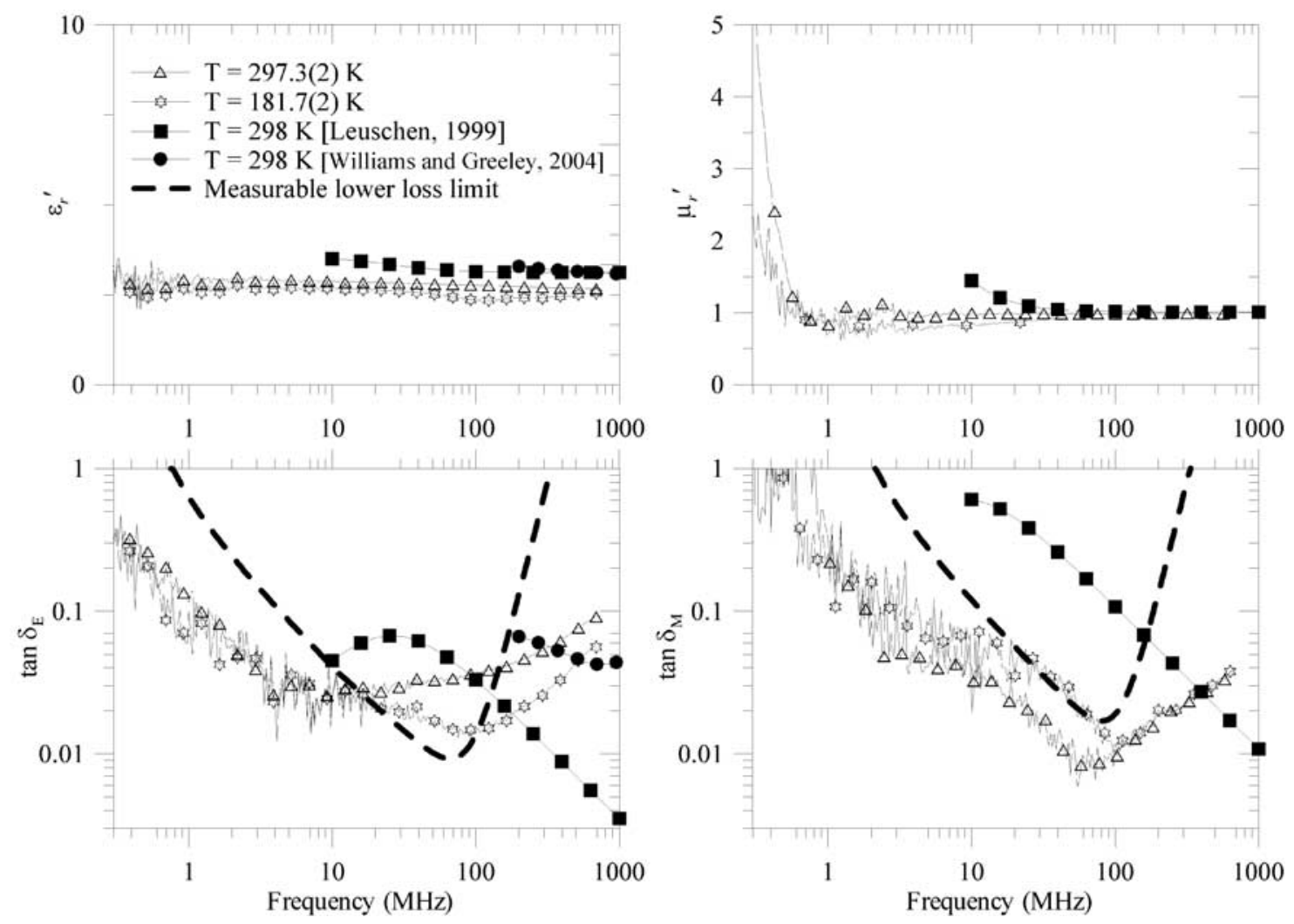

Figure 4. Broad temperature-dependent dielectric relaxation of JSC-1 along with measurements made by Leuschen [1999] and Williams and Greeley [2004]. Symbols are located at every tenth data point for data collected in this research. Symbols are located at every tenth data point. Measurement errors are much smaller than the symbols, except for the portion of the data that is below the measurable lower loss limit and in the $\mu_{r}^{\prime}$ at frequencies below $1 \mathrm{MHz}$. Uncertainties for the other EM property measurements were not given.

inside a duplicate sample holder that was packed with the same material and placed next to the actual test sample holder. A VNA measurement was taken once the temperature inside the duplicate sample holder remained within $\pm 0.1 \mathrm{~K}$ of the target temperature for approximately 15 minutes. Since the temperature inside the actual test sample holder could not be measured, the temperature of the measurement was determined by averaging the temperature measurements of the duplicate sample holder for 15 minutes prior to the measurement and for four minutes during the measurement. If a Martian analog sample was found to have temperature and/or frequency-dependent EM properties, the data were inverted using MATLAB ${ }^{\mathbb{R}}$ 's optimization toolbox (version 2.2) inversion to find the Cole-Cole and generalized Boltzmann parameters [Stillman, 2006].

\section{Measurement Results}

[36] Five of the twelve measured samples have no measurable EM losses; therefore they are frequency independent and have no imaginary components (Tables 1 and 2). The EM properties of jarosite are shown as a representative sample of this group of data (Figure 1).

[37] Four (GH-1, GH-2, JSC-1 and Plag) of the twelve measured samples have temperature-dependent dielectric relaxation losses (Figures 2-5). Their Cole-Cole and Boltzmann parameters are listed in Tables 2 and 3. An Arrhenius plot of the GH-1 data shows how the frequency dependence changes with temperature (Figure 6). Only temperature data in the range of 181-227 K were used to determine the Boltzmann parameters because at higher temperatures the relaxation was too close to the resonant frequency of the sample holder.

[38] Three (Mag-1, Mag-2, Mag-3) of the twelve Martian analog samples have temperature-independent magnetic relaxation losses (Figure 7). The magnetic relaxations have a narrow distribution of time constants of relaxation (high $\alpha$ ) (Tables 4 and 5). This means that there are few variations in the mechanism that cause the magnetic relaxation.

\section{Discussion}

\subsection{Hematite}

[39] Both gray hematite samples (GH-1 and GH-2) were found to have a temperature-dependent dielectric relaxation and a temperature- and frequency-independent magnetic permeability. The GH-1 sample is composed largely of gray hematite and has a dielectric relaxation centered at $1.42 \mathrm{GHz}$ at room temperature $(298 \mathrm{~K})$ and $230 \mathrm{MHz}$ at the average Martian temperature $(213 \mathrm{~K})$. The GH-2 sample is composed of $65 \%$ gray hematite and $10 \%$ magnetite and has a dielectric relaxation centered at $2.52 \mathrm{GHz}$ at room temperature $(298 \mathrm{~K})$ and $450 \mathrm{MHz}$ at the average Martian temperature $(213 \mathrm{~K})$. The temperature dependence of GH-1 


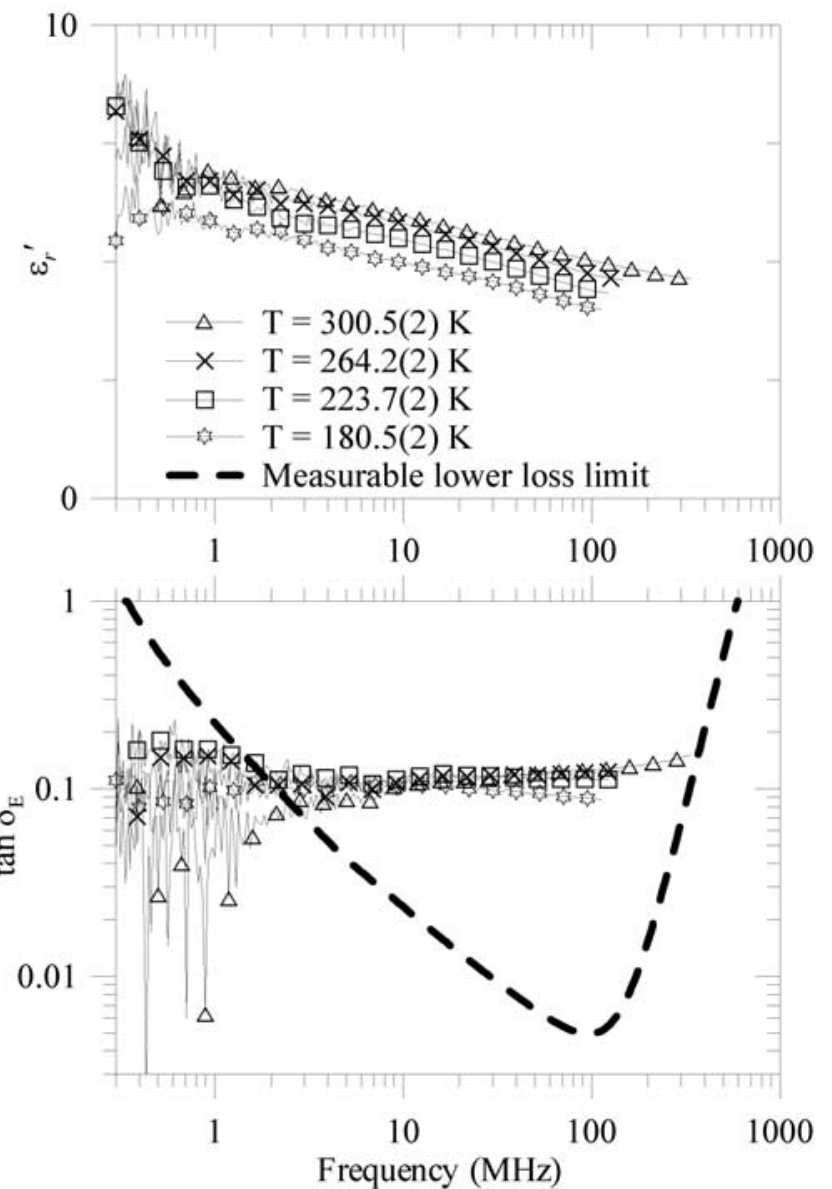

Figure 5. Broad temperature-dependent dielectric relaxation of Plag. Measurement errors are much smaller than the symbols, except for the portion of the data that is below the measurable lower loss limit.

creates a factor of $\approx 30$ change in the attenuation rate at MARSIS and SHARAD frequencies over the entire Martian temperature range (Figure 8). This demonstrates why EM property measurements for Mars analogs must be made at Martian temperatures.

[40] The temperature-dependent dielectric relaxation of gray hematite is believed to be caused by a molecular polarization mechanism because of its rhombohedra-corundum crystal structure. This crystal structure creates parallel planes of cation and anions. The application of an external electric field causes the hematite crystal to distort by shifting the planes of cations and anions in opposite directions, thus creating charge separation. As the temperature is decreased, the cations and anions have less energy and can no longer polarize as quickly. The activation energy describes how quickly this shift occurs with temperature, and represents the energy barrier that the charges must overcome in order to become polarized. The gray hematite samples, GH-1 and GH-2, have activation energies of $0.1434( \pm 0.0023) \mathrm{eV}$ and $0.134(+0.009,-0.007) \mathrm{eV}$, respectively.

[41] However, red hematite has the same chemical formula and the same unit cell crystal structure as gray hematite. EM measurements of red hematite determined that it does not have a molecular polarization mechanism at radar frequencies $(1-1000 \mathrm{MHz})$. The difference between the two hematites is that red hematite is composed of randomly oriented unit cell crystals, while gray hematite is composed of densely packed and aligned unit cell crystals that are bonded together to form a coarse grained crystal. The coarse grained crystal possesses parallel sheets of $\mathrm{Fe}$ cations and $\mathrm{O}$ anions. When an electric field is induced these sheets move toward each other, thus creating a molecular polarization. Red hematite does not contain any sheets of cations or anions and therefore no molecular polarization was observed.

\subsection{Magnetite}

[42] The three magnetite samples (Mag-1, Mag-2, Mag-3) measured in this study have a temperature-independent magnetic relaxation with relaxation frequencies that vary from 177-884 MHz (Figure 7). The magnetic relaxations all have poorly constrained high-frequency limits, as the relaxation frequency is greater than the resonant frequency of the sample holder. Magnetic relaxations were not measured in the gray hematite sample, GH-2, which contains a minor amount $(10 \%)$ of magnetite and has a magnetic permeability of $1.22( \pm 0.03)$. However, any magnetic relaxations occurring with a frequency greater than $400 \mathrm{MHz}$ would not have been detected because of resonance. JSC Mars-1 also contains some magnetite but does not possess any magnetic losses at radar frequencies, most likely

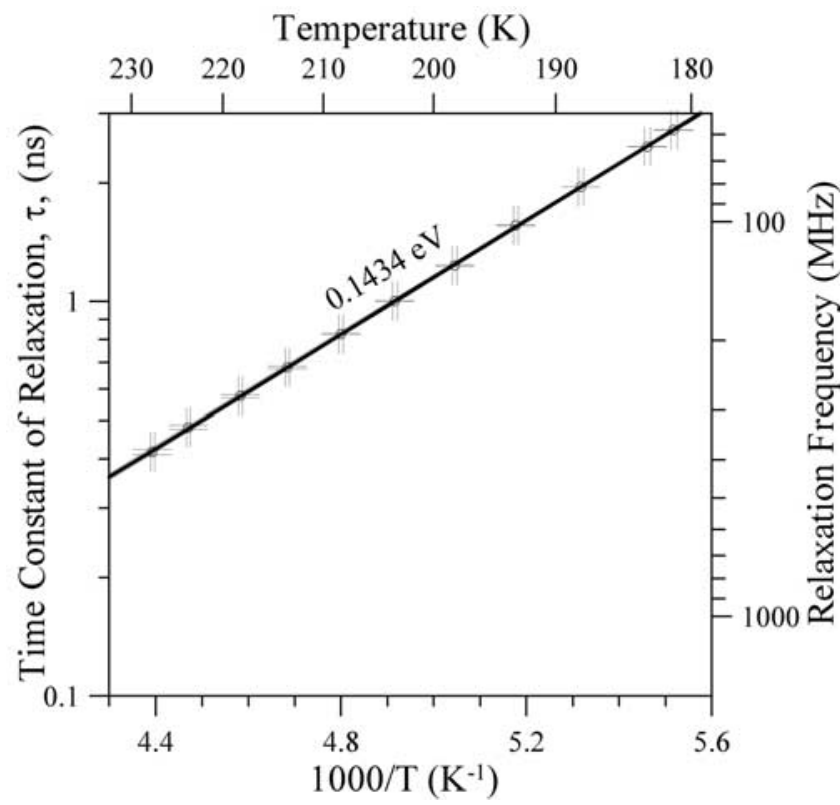

Figure 6. Arrhenius plot of GH-1. The dark black line is the best fit line of data points with a temperature range from 181 to $227 \mathrm{~K}$ (black data points). The two light black lines are the boundaries of the minimum and maximum best fit. Only data from the coldest temperature measurements are shown since the relaxation shifted outside the range of usable data as the temperature increased. The error bars show the $95.5 \%$ confidence intervals of the time constant of relaxation (vertical) and temperature (horizontal). 
Table 3. Cole-Cole and Boltzmann Temperature Parameters for Samples With Temperature-Dependent Dielectric Relaxations and Frequency-Independent Magnetic Permeability ${ }^{a}$

\begin{tabular}{|c|c|c|c|c|c|c|c|c|}
\hline Sample & Density (g/cc) & $\varepsilon_{\mathrm{DC}}$ & $\varepsilon_{\infty}$ & $\tau_{\infty}(\mathrm{ns})$ & $\mathrm{E}(\mathrm{eV})$ & $\alpha$ & $\sigma_{\mathrm{DC}}(\Omega \mathrm{km})$ & $\mu_{\mathrm{r}}$ \\
\hline \multirow{2}{*}{ GH-1 } & 3.11 & $27.24(21)$ & $6.61(18)$ & $2.811 \times 10^{-4}$ & 0.1434 & $0.843(10)$ & $>15$ & $1.00(2)$ \\
\hline & 1.60 & $10.17(8)$ & $2.47(7)$ & $2.811 \times 10^{-4}$ & 0.1434 & $0.843(10)$ & $>15$ & $1.00(2)$ \\
\hline \multirow[t]{2}{*}{ GH-2 } & 2.40 & $17.0(7)$ & $4.9(3)$ & $2.33 \times 10^{-4}$ & 0.134 & $0.55(+14,-8)$ & $>15$ & $1.22(3)$ \\
\hline & 1.60 & $10.1(4)$ & $2.9(2)$ & $2.33 \times 10^{-4}$ & 0.134 & $0.55(+14,-8)$ & $>15$ & $1.13(3)$ \\
\hline \multirow[t]{2}{*}{ Plag } & 1.59 & $8.4(0.5)$ & 2.84 & 0.0863 & 0.111 & $0.291(16)$ & $>15$ & $1.00(2)$ \\
\hline & 1.60 & $8.4(0.5)$ & 2.84 & 0.0863 & 0.111 & $0.291(16)$ & $>15$ & $1.00(2)$ \\
\hline \multirow[t]{2}{*}{ JSC-1 } & 0.90 & $3.4(10)$ & 1.80 & $9.3 \times 10^{-5}$ & 0.175 & $0.13(+1,-5)$ & $>15$ & $1.00(2)$ \\
\hline & 1.60 & $5.3(10)$ & 2.84 & $9.3 \times 10^{-5}$ & 0.175 & $0.13(+1,-5)$ & $>15$ & $1.00(2)$ \\
\hline
\end{tabular}

${ }^{\mathrm{a}}$ Uncertainties in the time constant of relaxation at infinite temperature, $\tau_{\infty}(\mathrm{ns})$, and activation energy, $\mathrm{E}(\mathrm{eV})$, are given in Table 4 . The first row shows the modeled results of the measured data. In the second row, the modeled data were corrected for density using a Lichtenecker power law mixing formula for the dielectric permittivity (equation (8)) and using equation (9) for the magnetic permeability. Values in parenthesis indicate the uncertainty in the last $\operatorname{digit}(\mathrm{s})$.

because of its titanium content. Low-frequency measurements using Sapphire Instruments and Bartington Instruments magnetic susceptibility meters measured a $\mu_{D C}^{\prime}=$

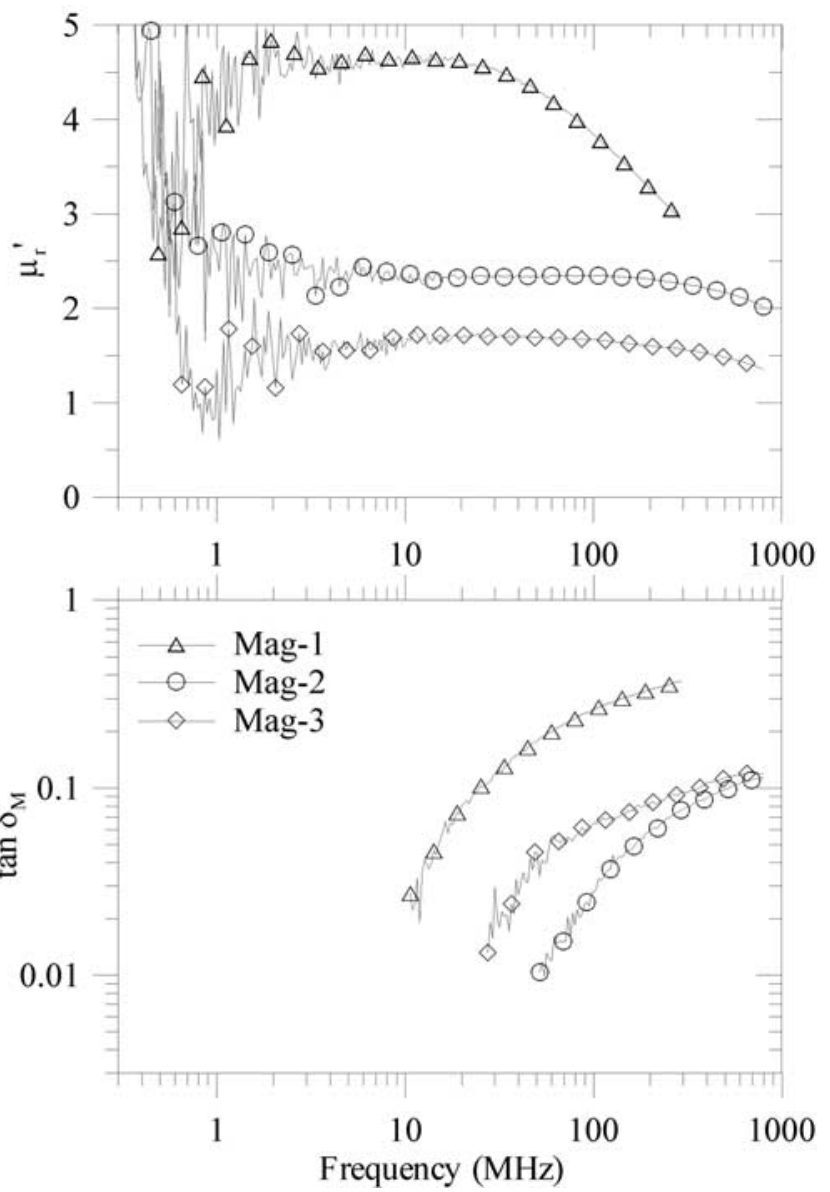

Figure 7. Magnetic relaxation of Mag-1, Mag-2, and Mag-3. Only the data above the measurable lower loss limit are shown. (The measurable lower loss limit is not shown since it varies for each sample.) The Mag-3 results fall within the error bounds of the results of Olhoeft and Capron [1993, 1994].
1.00783 for JSC-1 (Figure 9). The relaxation frequency was poorly constrained to greater than $6 \mathrm{kHz}$ and less than $600 \mathrm{kHz}$, as only two real magnetic permeability data points were measured on the relaxation. However, such a relaxation could only produce a maximum magnetic loss tangent of 0.004. Magnetite also has a frequency-independent dielectric permittivity that is greater than its predicted density derived electronic polarization value. This indicates that magnetite has a dielectric relaxation at frequencies greater than $800 \mathrm{MHz}$.

[43] The magnetic relaxations observed in magnetite samples in this study are believed to be caused by magnetic domain wall displacement. Evidence for this mechanism includes the following: the relaxations were temperature independent, the frequency of the relaxation ranged from $177-884 \mathrm{MHz}$, and the magnetite has multidomain grains. While magnetic domains can change as a function of temperature [Dunlop and Özdemir, 1997], the magnetic permeability measurements in this study were only sensitive to the way magnetic domains move in response to an external magnetic field as a function of temperature. The measurements in this study show that magnetic domain displacement is not sensitive to temperature from $180-300 \mathrm{~K}$. However, none of the samples were measured near their Curie temperature where magnetic domain displacement may be sensitive to temperature. Above the Curie temperature, no magnetic

Table 4. Uncertainties in the Time Constant of Relaxation at Infinite Temperature, $\tau_{\infty}$ (ns), and Activation Energy, E (eV), for Selected Samples With Dielectric Permittivity Relaxations

\begin{tabular}{llll}
\hline Sample & Temperature Range & \multicolumn{1}{c}{$\tau_{\infty}$} & \multicolumn{1}{c}{$\mathrm{E}$} \\
\hline GH-1 & $>227 \mathrm{~K}$ & $2.81(+39,-35) \times 10^{-4}$ & $0.1434(23)$ \\
& $181-227 \mathrm{~K}$ & $2.81(15) \times 10^{-4}$ & $0.1434(7)$ \\
& $<181 \mathrm{~K}$ & $2.81(+35,-39) \times 10^{-4}$ & $0.1434(23)$ \\
GH-2 & $>213 \mathrm{~K}$ & $2.3(+12,-10) \times 10^{-4}$ & $0.134(+9,-7)$ \\
& $184-213 \mathrm{~K}$ & $2.3(+5,-7) \times 10^{-4}$ & $0.134(+5,-3)$ \\
& $<184 \mathrm{~K}$ & $2.3(+10,-12) \times 10^{-4}$ & $0.134(+7,-9)$ \\
Plag & $303-180 \mathrm{~K}$ & $0.0863(+28,-8)$ & $0.111(5)$ \\
JSC-1 & $303-180 \mathrm{~K}$ & $9.3(+14.8,-5.9) \times 10^{-5}$ & $0.175(21)$ \\
\hline
\end{tabular}


Table 5. Cole-Cole Parameters for Samples With Temperature-Independent Magnetic Relaxations and Frequency-Independent Dielectric Permittivity $^{\mathrm{a}}$

\begin{tabular}{lcllllll}
\hline Sample & Density $(\mathrm{g} / \mathrm{cc})$ & \multicolumn{1}{c}{$\varepsilon_{\mathrm{r}}^{\prime}$} & \multicolumn{1}{c}{$\mu_{\mathrm{DC}}$} & \multicolumn{1}{c}{$\mu_{\infty}$} & $\tau(\mathrm{ns})$ & $\alpha$ \\
\hline Mag-1 & 2.56 & $10.61(11)$ & $4.89(+5,-12)$ & $1.66(+23,-17)$ & $0.80(+1,-7)$ & $0.76(+4,-5)$ & $\sigma_{\mathrm{DC}}(\mathrm{k} \Omega \mathrm{m})$ \\
& 1.60 & $5.67(6)$ & $1.73(+2,-4)$ & $1.29(+18,-13)$ & $0.80(+1,-7)$ & $0.76(+4,-5)$ & $>15$ \\
Mag-2 & 1.88 & $6.92(10)$ & $2.35(2)$ & $1.93(+18,-25)$ & $0.28(+13,-7)$ & 1.00 & $>15$ \\
& 1.60 & $5.76(8)$ & $1.93(2)$ & $1.68(+16,-22)$ & $0.28(+13,-7)$ & 1.00 & $>15$ \\
Mag-3 & 2.23 & $6.82(10)$ & $1.73(+1,-3)$ & $1.24(24)$ & $0.30(+20,-12)$ & $0.75(+10,-2)$ & $>15$ \\
& 1.60 & $4.52(7)$ & $1.41(+1,-2)$ & $1.16(22)$ & $0.30(+20,-12)$ & $0.75(+10,-2)$ & $>15$ \\
\hline
\end{tabular}

${ }^{\mathrm{a}}$ The first row shows the modeled results of the measured data. In the second row, the modeled data were corrected for density using a Lichtenecker power law mixing formula for the dielectric permittivity (equation (8)) and using equation (9) for the magnetic permeability assuming magnetite is at a concentration of a $100 \%$ when measured.

relaxations could exist because the low-frequency limit of the relative magnetic permeability would be one. Therefore magnetic relaxations must be temperature dependent near the Curie temperature of the mineral. The Curie temperature of a titanomagnetite, $\mathrm{Fe}_{(2-2 \mathrm{x})}^{3+} \mathrm{Fe}_{(1+\mathrm{x})}^{2+} \mathrm{Ti}_{\mathrm{x}}^{4+} \mathrm{O}_{4}^{2-}$ where $\mathrm{x}$ is the mole $\%$ of the titanium impurity, is dependent on the amount of titanium impurities [Hunt et al., 1995]. At titanium concentrations greater than $\mathrm{x}=0.77$, the Curie temperature of titanomagnetite is in the Martian temperature range [Hunt et al., 1995]. In addition, according to the Néel model, the activation energy is a function of particle volume, saturation magnetization, and coercivity. This research found that the activation energy of magnetite must be smaller than $0.03 \mathrm{eV}$, which is the smallest activation that can be measured for magnetic permeability with the measurement apparatus used in this research.

[44] The Mag-1 and the Mag-2 samples are composed of almost entirely magnetite. The Mag- 1 sample has a $\mu_{D C}^{\prime}=$ 4.9, while the Mag-2 sample has a $\mu_{D C}^{\prime}=2.3$. We believe that the reduced strength of Mag- 2 may be due to the

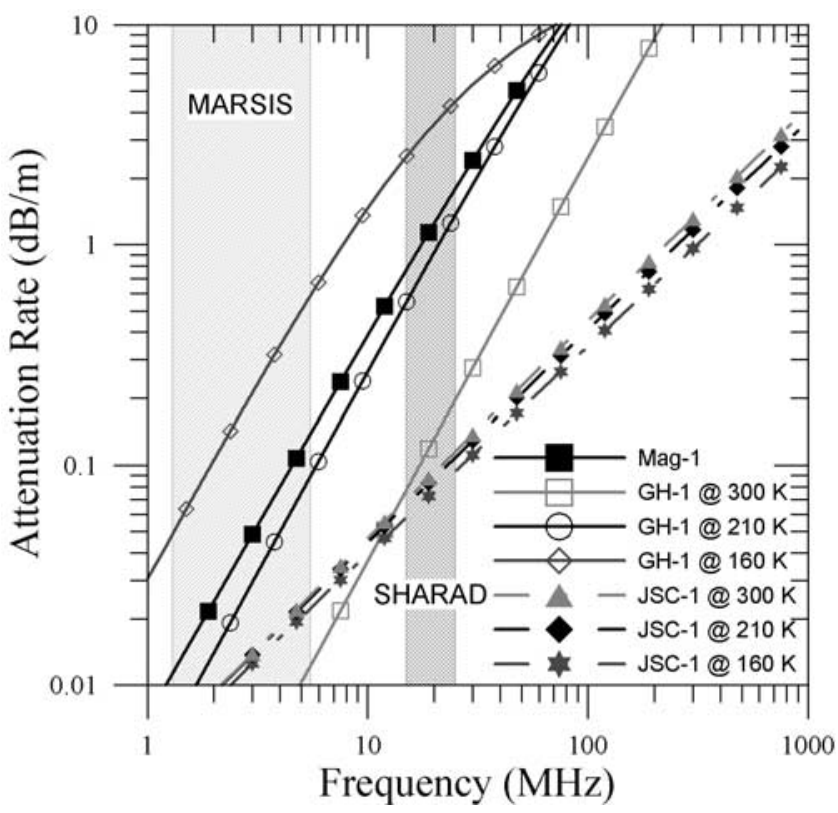

Figure 8. Attenuation rate versus frequency of magnetite, gray hematite, and absorbed water (JSC-1) at the measured density of the samples. presence of greater titanium impurities and/or a slight oxidation of some of the magnetite to maghemite and not due to any grain size effects since the grain size is considerably larger than the critical single domain size of magnetite. The Mag-3 sample has a sand component in addition to magnetite. Therefore the Mag-3 sample has the lowest magnetic losses of the three magnetic samples.

\subsection{Plagioclase}

[45] JSC-1 and Plag samples have a small, broad, dielectric relaxation. Two previous studies [Leuschen, 1999; Williams and Greeley, 2004] have measured the EM properties of JSC-1 at radar frequencies (Figure 4). It appears that both Leuschen [1999] and Williams and Greeley [2004] measured a JSC-1 sample that was slightly more dense and/ or contained slightly more water than the sample measured in this research, as their real part of the relative dielectric permittivity is slightly greater than those measured in this study. However, neither of the previous studies report sample density. The measurements made by Williams and Greeley [2004] are also greater in the loss tangent than those measured in this study. This can also be explained by a slightly greater density and/or water content. The measurements made by Leuschen [1999] do not correspond with either data set. This is most likely because the data were fit with a Debye relaxation instead of a broad Cole-Cole relaxation. Leuschen [1999] magnetic measurements also do not correspond with data acquired in this study.

[46] The JSC-1 and Plag relaxations were difficult to model since neither the low- nor high-frequency limits could be measured. Consequently, the high-frequency limit was assumed to have a real part of the relative dielectric permittivity of 2.84 , which is the density controlled electronic polarization value as determined by a Lichtenecker

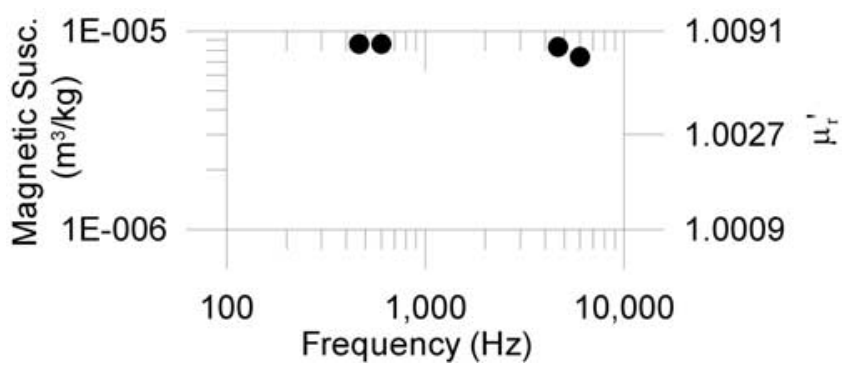

Figure 9. Magnetic relaxation of JSC Mars-1. 
power law mixing formula. While the models are poorly constrained, it is important to note that these models produce complex dielectric permittivity values that mimic the data from $1-1000 \mathrm{MHz}$ over a temperature range from $180-298 \mathrm{~K}$.

[47] We believe that interfacial polarization is the mechanism causing the broad temperature-dependent dielectric relaxation loss in these plagioclase rich samples. Although these samples where vacuumed dried for days, we believe that this relaxation is caused by absorbed water. The strength of the bond between the water and soil makes absorbed water behave much differently than free water. The differences include the following: vacuum cannot remove all absorbed water, the freezing point of absorbed water is significantly depressed, the relaxation frequency of absorbed water is lower and more distributed (low $\alpha$ ) [Hasted, 1973]. The broad distribution of the relaxation creates a low attenuation rate over a broad range of frequencies (Figure 8).

\subsection{Other Samples}

[48] The maghemite (Maghem) sample measured in this study has a frequency-independent magnetic permeability of $1.28 \pm 0.02$ at radar frequencies. However, the magnetic permeability high-frequency limit must relax to a value of one at frequencies greater than $1 \mathrm{GHz}$ (highest frequency measured with this sample). Other Martian analogs measured in this study including red hematite, jarosite, and olivine, all of which have no measurable EM losses. Thus GPR depth of penetration on Mars will likely be limited by other factors such as scattering losses where the subsurface is composed largely of these minerals.

\section{Conclusions}

[49] The measurements conducted in this study characterized several significant Martian EM relaxations in Martian analog minerals and found that dielectric relaxations of Martian analogs do vary as a function of temperature. Therefore lab measurements of EM properties must be made at Martian temperatures. The attenuation rate at room temperature $(298 \mathrm{~K})$ and at Martian average temperature $(213 \mathrm{~K})$ differs by a factor of $\approx 7$ for GH-1 (Figure 8 ). The magnetic relaxations that were measured in this study did not vary as a function of temperature, and therefore must have a small $(<0.03 \mathrm{eV})$ activation energy.

[50] The only magnetic relaxations that can significantly affect GPR are possessed by ferromagnetic and ferrimagnetic minerals. This is because they are the only type of magnetic materials that can have a magnetic permeability greater than 1.05. Magnetic relaxations were also found to have narrow (high $\alpha$ ) relaxations. This is important because it implies that magnetic relaxations will only cause attenuation over a narrow frequency range. These magnetic relaxations were found to only occur at relatively high frequencies of less than $200 \mathrm{MHz}$. Lastly, because magnetic properties mix nonlinearly, the magnetic permeability is significantly reduced as the concentration of magnetite decreases. Therefore small concentrations of magnetite will not cause significant GPR attenuation. Consequently, EM energy at GPR frequencies should not be significantly attenuated through the Martian global magnetic dust layer.
[51] Not all of Mars will be composed of minerals that have large dielectric or magnetic relaxation losses. In fact, the most significant loss on Mars may be from absorbed water. The strength of the absorbed water relaxation will be related to the surface area (i.e., how much water can be absorbed) of the subsurface. JSC Mars-1 has a surface area of $85.1 \mathrm{~m}^{2} / \mathrm{g}$ [Grimm et al., 2007], which is larger than Viking's determination of Martian dust of $17 \mathrm{~m}^{2} / \mathrm{g}$ [Ballou et al., 1978]. While the Martian dust may not be able to hold much absorbed water, high-surface area clays (i.e., montmorillonite and smectites) can hold significant amounts of absorbed water, and thus could cause significant radar attenuation.

\section{Notation}

c velocity of an EM wave in vacuum, $2.99792458 \times$ $10^{8} \mathrm{~m} / \mathrm{s}$.

$d$ bulk density, $\mathrm{g} / \mathrm{cm}^{3}$.

$d_{n}$ normalized bulk density $1.60 \mathrm{~g} / \mathrm{cm}^{3}$.

$f_{r}$ relaxation frequency, $\mathrm{Hz}=1 / \mathrm{s}$.

$H_{c}$ coercivity, $\mathrm{A} / \mathrm{m}$.

$J_{s}$ magnetic saturation normalized by density, $\mathrm{Am}^{2} / \mathrm{kg}$.

$M_{s}$ saturation magnetization, $\mathrm{A} / \mathrm{m}$.

$k$ Boltzmann constant, $8.6176 \times 10^{-5} \mathrm{eV} / \mathrm{K}$.

$k$ wave number, $1 / \mathrm{m}$.

$K$ mode of the high-frequency limit of 114 lunar samples, 261 pure minerals, and 367 rocks [Olhoeft and Strangway, 1975], $1.93 \pm 0.17$.

$M_{s}$ magnetic saturation, $\mathrm{A} / \mathrm{m}$.

$t$ time, $\mathrm{s}$.

$x$ mole $\%$ of the titanium in magnetite and maghemite percent.

$X$ relative dielectric permittivity or relative magnetic permeability.

$X_{\infty}$ infinite or high-frequency limit of $\mathrm{X}$.

$X_{D C}$ static, DC, or low-frequency limit of $\mathrm{X}$.

$\alpha$ attenuation coefficient, $\mathrm{Np} / \mathrm{m}$.

$\alpha$ Cole-Cole distribution parameter.

$\beta$ phase coefficient, radians $/ \mathrm{m}$.

$\delta_{D}$ dielectric loss tangent, radians.

$\delta_{C}$ conduction loss tangent, radians.

$\delta_{E}$ electrical loss tangent, radians.

$\delta_{M}$ magnetic loss tangent, radians.

$\delta_{E M}$ electromagnetic loss tangent, radians.

$\varepsilon_{o} \quad$ dielectric permittivity of free space, $8.854 \times 10^{-12} \mathrm{~F} /$ $\mathrm{m}$ or $\mathrm{s}^{4} \mathrm{~A}^{2} / \mathrm{m}^{3} \mathrm{~kg}$.

$\varepsilon_{r}^{*}$ complex relative dielectric permittivity.

$\varepsilon_{r}^{\prime}$ real part of the relative dielectric permittivity.

$\varepsilon_{r}^{\prime \prime}$ imaginary part of the relative dielectric permittivity.

$\varepsilon_{D C}$ static, DC, or low-frequency limit of the real part of the relative dielectric permittivity.

$\varepsilon_{\infty} \quad$ infinite or high-frequency limit of the real part of the relative dielectric permittivity

$\varepsilon_{1}^{*} \quad$ complex relative dielectric permittivity for material 1 .

$\varepsilon_{2}^{*}$ complex relative dielectric permittivity for material 2 .

$\varepsilon_{\mathrm{m}}^{*}$ complex relative dielectric permittivity predicted for mixture.

$\varepsilon_{r}^{* n}$ complex relative dielectric permittivity normalized to density.

$\eta$ attenuation of EM energy, dB. 
$\mu_{o} \quad$ magnetic permeability of free space, $1.256 \times 10^{-6}$ $\mathrm{H} / \mathrm{m}$ or $\mathrm{mkg} / \mathrm{s}^{2} \mathrm{~A}^{2}$

$\mu_{r}^{*}$ relative magnetic permeability.

$\mu_{r}^{\prime}$ real part of the relative magnetic permeability.

$\mu_{r}^{\prime \prime} \quad$ imaginary part of the relative magnetic permeability.

$\mu_{D C}$ static, DC, or low-frequency limit of the real part of the relative magnetic permeability.

$\mu_{\infty}$ infinite or high-frequency limit of the real part of the relative magnetic permeability.

$\sigma_{D C}$ static or DC electrical conductivity, $1 / \Omega \mathrm{m}$.

$\tau$ time constant of relaxation, $\mathrm{s}$.

$\tau_{\infty}$ time constant of relaxation at infinite temperature, $\mathrm{s}$.

$\Omega$ volume fraction of material 2 in the mixture, $\%$.

$\omega$ angular (radian) frequency, $\mathrm{Hz}=$ radians $/ \mathrm{s}$.

[52] Acknowledgments. We thank Steve Sutley (USGS-Denver) for conducting our XRD measurements, Al Modroo for collecting some of the samples, and Justin Modroo, Brianne Douthit, Beau Winters, Matt Hergert, Andy Kass, Sjoerd de Ridder, Ross Wagle, Paul Schwering, and Francis Li for providing experimentation assistance. This project was funded by NASA grant 20119458 NAG5-12754.

\section{References}

Adams, S. F. (1969), Microwave Theory and Applications, 513 pp., Prentice-Hall, Upper Saddle River, N. J.

Allen, C. C., and R. V. Morris (1999), Reply: Caution advised on suitability of a Mars soil simulant: Reply, Eos Trans. AGU, 80, 169.

Allen, C. C., et al. (1997), JSC Mars-1: Martian Regolith Simulant, paper presented at 28th Lunar and Planetary Science Conference, Houston, Texas, abstract \#1797, Lunar and Planetary Institute, 17-21 March.

Allen, C. C., et al. (1998a), Martian Regolith Simulant JSC Mars-1, paper presented at 29th Lunar and Planetary Science Conference, Houston, Texas, abstract \#1690, Lunar and Planetary Institute, 16-20 March.

Allen, C. C., et al. (1998b), Martian soil stimulant available for scientific, educational study, Eos Trans. $A G U, 79,405$.

Baker-Jarvis, J., et al. (1993), Transmission/reflection and short-circuit line methods for measuring permittivity and permeability, NIST Technical Note 1355-R, 112 pp., NIST, Boulder, CO.

Ballou, E. V., et al. (1978), Chemical interpretation of Viking Lander 1 life detection experiment, Nature, 217, 644.

Bertelsen, P., et al. (2004), Magnetic properties experiments on the Mars exploration rover Spirit at Gusev Crater, Science, 305, 827-829.

Bibring, J. P., et al. (2006), Global mineralogical and aqueous Mars history derived from OMEGA/Mars express data, Science, 312, 404.

Chernicoff, S., and R. Venkatakrishnan (1995), Geology, 593 pp., Worth Publishers, New York.

Christensen, P. R., et al. (2001), Global mapping of Martian hematite mineral deposits: Remnants of water-driven processes on early Mars, J. Geophys. Res., 106, 23,873-23,885.

Clifford, S. M. (1993), A model for the hydrologic and climatic behavior of water on Mars, J. Geophys. Res., 98, 10,973-11,016.

Cole, K. S., and R. H. Cole (1941), Dispersion and absorption in dielectrics, J. Chem. Phys., 9, 341-351.

Debye, P. (1929), Polar Molecules, 172 pp., Chemical Catalog, New York.

Dunlop, D. J., and J. Arkani-Hamed (2005), Magnetic minerals in the Martian crust, J. Geophys. Res., 110, E12S04, doi:10.1029/ 2005JE002404.

Dunlop, D. J.. and O. Özdemir (1997), Rock Magnetism Fundamentals and Frontiers, 583 pp., Cambridge Univ. Press, Cambridge, U.K.

Goetz, W., et al. (2005), Indication of drier periods on Mars from the chemistry and mineralogy of atmospheric dust, Nature, 436, 62-64.

Grimm, R. E., et al. (2006), Absorption and scattering in ground-penetrating radar: Analysis of the Bishop Tuff, J. Geophys. Res., 111, E06S02, doi:10.1029/2005JE002619.

Grimm, R. E., et al. (2007), Abundance and electrical properties of interfacial water in the Martian regolith, Lunar Planet. Sci., XXXVIII, Houston, TX, abstract \#2249, Lunar and Planetary Institute, 12-16 March.

Grimnes, S., and Ø. G. Martinsen (2000), Bioimpedance and Bioelectricity Basics, 359 pp., Academic Press, New York.

Hargraves, R. B., et al. (1979), Viking magnetic properties experiment: Extended mission results, J. Geophys. Res., 84, 8379-8384.

Hargraves, R. B., et al. (1999), Caution advised on suitability of a Mars soil simulant, Eos Trans. AGU, 80, 168-169.

Hargraves, R. B., et al. (2000), Magnetic enhancement on the surface of Mars?, J. Geophys. Res., 105, 1819-1827.
Hasted, J. B. (1973), Aqueous Dielectrics, pp. 136-165, Chapman and Hall, London.

Heggy, E., and A. Pommerol (2005), Dielectric Map of the Martian Surface, paper presented at Workshop on Radar Investigations, Lunar and Planet. Inst., Houston, Texas, abstract \#6028, Lunar and Planetary Institute, 7-10 February.

Heggy, E., et al. (2001), On water detection in the Martian subsurface using sounding radar, Icarus, 154, 244-257.

Heggy, E., et al. (2003), Local geoelectric models of the Martian subsurface for shallow groundwater detection using sounding radars, J. Geophys. Res., 108(E4), 8030, doi:10.1029/2002JE001871.

Hewlett-Packard (1994), HP8752C and HP8753D Network Analyzers: Technical Specifications, 35 pp., Hewlett-Packard, 5962-9770E.

Hoefen, T. M., et al. (2003), Discovery of olivine in the Nili Fossae region of Mars, Science, 302, 627-630.

Hunt, C. P., et al. (1995), Magnetic properties of rocks and minerals, in Rock Physics and Phase Relations, edited by T. J. Ahrens, pp. 189-204, AGU, Washington, D. C.

Iben, I. E. T. et al. (1996), Dielectric Properties of Soil: Application to Radio Frequency Ground Heating, 35 pp., GE Global Research, Report Number 96CRD150.

Kauzmann, W. (1942), Dielectric relaxation as a chemical rate process, Rev. Mod. Phys., 14, 12- 44.

Klingelhöfer, G., et al. (2004), Jarosite and hematite at Meridiani Planum from opportunity's Mössbauer spectrometer, Science, 306, 1740-1745.

Leuschen, C. (1999), Analysis of the complex permittivity and permeability of a Martian soil simulant from $10 \mathrm{MHz}$ to $1 \mathrm{GHz}$, paper presented at Proc. of the 1999 IEEE Geoscience and Remote Sensing Symposium, vol. 4, pp. 2264-2266, IEEE, 23 June-2 July, Hamburg, Germany.

Madsen, M. B., et al. (2005), An update on results from the magnetic properties experiments on the Mars exploration rovers spirit and opportunity, paper presented at 34th Lunar and Planetary Science Conference, Houston, TX, abstract \#2379, Lunar and Planetary Institute, 14-18 March.

McIntosh, R. L. (1966), Dielectric Behavior of Physically Adsorbed Gases, 160 pp., Marcel Dekker, New York.

Morris, R. V., D. C. Golden, and J. F. Bell III (1997), Low-temperature reflectivity spectra of red hematite and the color of Mars, J. Geophys. Res., 102, 9125-9133.

Morris, R. V., et al. (2001), Phyllosilicate-poor palagonitic dust from Mauna Kea Volcano (Hawaii): A mineralogical analogue for magnetic Martian dust?, J. Geophys. Res., 106, 5057-5083.

Morris, R. V., et al. (2004), Mineralogy at Gusev Crater from the Mössbauer spectrometer on the Spirit Rover, Science, 305, 833-836.

Néel, L. (1949), Théorie du traînage magnétique des ferromagnétiques en grains fins avec applications aux terres cuites, Ann. Géophys., 5, 99136.

Olhoeft, G. R. (1976), Electrical properties of rocks, in The Physics and Chemistry of Rocks and Minerals, edited by R. J. G. Strens, pp. $261-$ 278, Wiley, London.

Olhoeft, G. R., and D. E. Capron (1993), Laboratory measurements of the radio frequency electrical and magnetic properties of soils from Yuma, Arizona, Open File Rep. 93-701, 214 pp., U. S. G. S., Denver, Colo.

Olhoeft, G. R., and D. E. Capron (1994), Petrophysical causes of electromagnetic dispersion, paper presented at Proc. of the Fifth Int'l. Conf. on Ground Penetrating Radar, Kitchener, Ontario, pp. 145-152.

Olhoeft, G. R., and D. W. Strangway (1974), Electrical properties of the surface layers of Mars, Geophys. Res. Lett., 1, 141-143.

Olhoeft, G. R., and D. W. Strangway (1975), Electrical properties of the first 100 meters of the moon, Earth Planet. Sci. Lett., 24, 394-404.

Pettinelli, E., et al. (2005), Laboratory investigations into the electromagnetic properties of magnetite/silica mixtures as Martian soil simulants, J. Geophys. Res., 110, E04013, doi:10.1029/2004JE002375.

Picardi, G., et al. (2005), Radar soundings of the subsurface of Mars, Science, 310, 1925-1928, doi:10.1126/science.1122165.

Safaeinili, A., et al. (2001), Radar Sounding of Mars: A Focus on MARSIS, paper presented at Conference on the Geophysical Detection of Subsurface Water on Mars, abstract \#7032, Lunar and Planetary Institute, 6-10 August.

Seu, R., et al. (2004), SHARAD: The MRO 2005 shallow radar, Planet. Space Sci., 52, 157-166.

Seu, R., et al. (2007), SHARAD sounding radar on the Mars Reconnaissance Orbiter, J. Geophys. Res., 112, E05S05, doi:10.1029/2006JE002745.

Stillman, D. E. (2006), Frequency and temperature dependence in electromagnetic properties of martian analog minerals [DVD-ROM], Ph.D. thesis, 199 pp. +, Colorado School of Mines, Golden, Colo.

Strangway, D. W. (1967), Magnetic characteristics of rocks, in Mining Geophysics, vol. 2, edited by D. A. Hansen et al., pp. 454-473, Soc. of Explor. Geophys., Tulsa, Okla. 
Squyres, S. W., and A. H. Knoll (2005), Sedimentary rock at Meridiani Planum: Origin, diagenesis, and implications for life on Mars, Earth Planet. Sci. Lett., 240, 1-10.

Ward, S. H., and G. W. Hohmann (1988), Electromagnetic theory for geophysical applications, in Electromagnetic Methods in Applied Geophysics, vol. 1, Theory, edited by M. N. Nabighian, pp. 131-312, Soc. of Explor. Geophys., Tulsa, Okla.

Williams, K. K., and R. Greeley (2004), Measurements of dielectric loss factors due to a Martian dust analog, J. Geophys. Res., 109, E10006, doi:10.1029/2002JE001957.

Wyatt, M. B., and H. Y. McSween Jr. (2002), Spectral evidence for weathered basalt as an alternative to andesite in the northern lowlands of Mars, Nature, 417, 263-266.
Yen, A. S., et al. (2005), An integrated view of the chemistry and mineralogy of Martian soils, Nature, 436, 49-53.

G. Olhoeft, Department of Geophysics, Colorado School of Mines, 1500 Illinois Street, Golden, CO 80401, USA. (golhoeft@mines.edu)

D. Stillman, Department of Space Studies, Southwest Research Institute, 1050 Walnut Street \#400, Boulder, CO 80302, USA. (dstillman@boulder. swri.edu) 